\title{
Analysis of Contact Force Variation between Contact Wire and Pantograph Based on Multibody Dynamics*
}

\author{
Mohd Azman ABDULLAH**, Yohei MICHITSUJI***, Masao NAGAI** \\ and Naoki MIYAJIMA** \\ **Department of Mechanical Systems Engineering, Tokyo University of Agriculture and Technology, \\ 2-24-16 Naka-cho, Koganei-shi, Tokyo, 184-8588, Japan \\ E-mail: mohdazman@utem.edu.my \\ *** Depatment of Mechanical Engineering, Ibaraki University \\ 4-12-1 Nakanarusawa-cho, Hitachi-shi, Ibaraki, 316-8511, Japan
}

\begin{abstract}
In recent years, high speed railway vehicle technologies have been studied and investigated in order to develop their performances with the objectives of improving riding comfort, noise reduction and high efficiency from environmental perspective. One of the essential performances is current collection stability. Operating at high speed conditions, the current collection system which consists of contact wire and pantograph suffers from contact force variation. In order to reduce such variation, pantograph design should be comprised by active control thus realizing stability. Numerical analysis can be applied to model the current collection and in particular simulate the contact force variation itself. In this study, Finite Element Method and Absolute Nodal Coordinate Formulation are used to model the wire. A free vibration experiment that focuses on the tension of wire is performed to validate the numerical models. The validated result from the experiment demonstrates the accuracy of the numerical analysis in modeling the wire. The techniques are then applied to model the contact wire for the contact force investigation. The result indicated the availability of pantograph design via simulation to achieve steady contact force.
\end{abstract}

Key words: Parameter Identification, Pantograph, Contact Wire, Absolute Nodal Coordinate Formulation, Multibody Dynamics

\section{Introduction}

Recently, a variety of railway technologies have been researched and developed in order to meet various needs in the railway transportation system. The main objectives are ensuring and improving passengers' comfort, making the system environmentally friendly and realizing high speed operation. Various measures such as active suspension system, low-noise pantograph and high-capacity drive system have been well developed and practically applied in order to meet the requirements. However, the stability of current collection system is hardly considered for high-speed operation. It is one of the important elements in the railway vehicle system to secure the stability of the power supply. Without a stable power supply, the train will not perform satisfactorily. At high speed operation, the bounce of pantograph due to the change in contact force with overhead power line causes instability. In order to obtain a stable contact force, parameter optimization and active control design are supposed to be effective counter measures. The past development of pantograph system was subjected to large-scale experimental facility with an actual size train ${ }^{(1)}$. It was constrained by cost and time. Therefore, development via numerical analysis 
and computer model simulation is in demand. And for that reason, detail model of overhead wire and pantograph system is necessary.

In the previous dynamic analysis approach, the springs are used to model the interactions ${ }^{(2)(3)(4)}$. This approach is too simple and not effective for the effect of the nonlinear deformation of the cable. Some analyses use partial differential equations to model the overhead wire as a continuous string or wire ${ }^{(5)(6)(7)}$. While partial differential equations are applicable for wave propagations study, they are not suitable for multibody dynamics. The use of linear finite element formulation in modeling the overhead wire ${ }^{(8)}$ does not account for the coupling effects and nonlinearities. Active control approaches via simulation have also been investigated previously ${ }^{(9)}$. While the modeling technique seemed appealing, the validation is feeble and not supported by any experimental result. Comparison between actual data from a running train and simulation has also been studied ${ }^{(10)}$. However, this study needs actual data which is not freely available due to confidentiality of the running train data from railway's company. Furthermore, the data is limited with certain designs and parameters.

In this study, we propose a flexible multibody dynamics analysis approach to model the overhead wires using Absolute Nodal Coordinate Formulation (ANCF) ${ }^{(10)(11)(12)(13)(14)}$. A simple free vibration of a wire experiment is conducted which focused on the tension of the overhead wires and unknown parameters identification. Three modeling techniques: classical Finite Element Method (FEM), ANCF and improved ANCF are compared by way of experiment for validation. The techniques are then applied to model overhead wire and pantograph interaction. The results show the potential of modeling via numerical analysis.

\section{Contact Wire Analysis Technique}

Three analysis techniques will be used and compared to model the stringing wire: classical FEM, ANCF and damping force accompanied ANCF (improved ANCF). The improved ANCF is the new technique proposed to enhance the ordinary ANCF. Figure 1 illustrates the elements of the wire. $O A$ and $O^{\prime} A$ ' elements represent the wire condition before and after deformation.

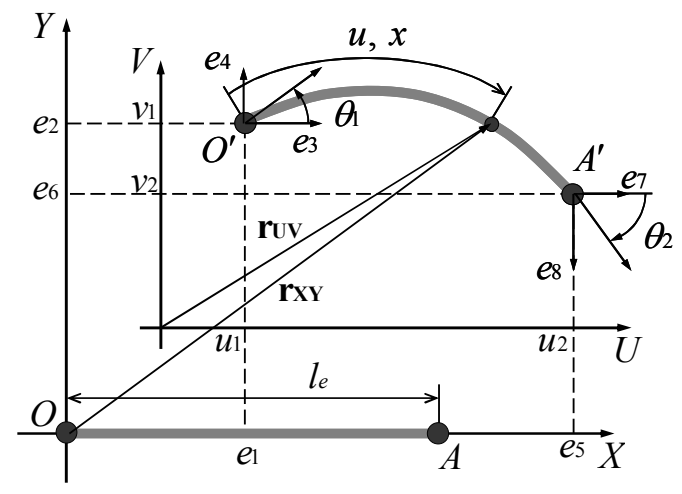

Fig. 1 Elements of wire

\subsection{Classical FEM}

The arbitrary element vector, $\mathbf{e}$ with local coordinate system $U V$ is shown as,

$\mathbf{e}=\left[\begin{array}{llllll}u_{1} & v_{1} & \theta_{1} & u_{2} & v_{2} & \theta_{2}\end{array}\right]^{T}$

And the position vector, $\mathbf{r}_{\mathbf{u v}}$ with shape function, $\mathbf{S}$, is represented by, 
$\mathbf{r u v}=\left[\begin{array}{l}\mathbf{S}_{1} \\ \mathbf{S}_{2}\end{array}\right] \mathbf{e}=\mathbf{S e}$

Here, $u_{1}, v_{1}$ is the $U V$ coordinate and $\theta_{1}$ the rotation angle of point $O$ '. Similarly, $u_{2}, v_{2}, \theta_{2}$ are the $U V$ coordinate and rotation angle of point $A^{\prime}$. The deformation theory of geometric stiffness and rigidity matrix are shown as,

$\mathbf{K}_{\varepsilon}=E a \int_{0}^{l_{e}}\left(\frac{\partial \mathbf{S}_{1}}{\partial u}\right)^{2} d u+E I \int_{0}^{l_{e}}\left(\frac{\partial^{2} \mathbf{S}_{2}}{\partial u^{2}}\right)^{2} d u$

$\mathbf{K}_{\eta}=\int_{V}\left(\left(\frac{\partial \mathbf{S}_{\mathbf{1}}}{\partial u}\right)^{T} \frac{P}{a}\left(\frac{\partial \mathbf{S}_{\mathbf{1}}}{\partial u}\right)+\left(\frac{\partial \mathbf{S}_{\mathbf{2}}}{\partial u}\right)^{T} \frac{P}{a}\left(\frac{\partial \mathbf{S}_{\mathbf{2}}}{\partial u}\right)\right) d V$

Here, $P$ is the axial power. Therefore, classical FEM equation of motion would be as follows,

$\mathbf{M e ̈}=\mathbf{Q}-\left(\mathbf{K}_{\varepsilon}+\mathbf{K}_{\eta}\right) \mathbf{e}$

And $\mathbf{Q}$ is an external force term such as gravity.

\subsection{ANCF}

At any point, the element vector e with absolute coordinate system $X Y$ is shown as,

$\mathbf{e}=\left[\begin{array}{llllllll}e_{1} & e_{2} & e_{3} & e_{4} & e_{5} & e_{6} & e_{7} & e_{8}\end{array}\right]^{T}$

And the position vector, $\mathbf{r}_{\mathrm{xy}}$ with shape function, $\mathbf{S}$, is represented by,

$$
\mathbf{r X Y}_{\mathbf{X Y}}=\left[\begin{array}{l}
\mathbf{S}_{1} \\
\mathbf{S}_{2}
\end{array}\right] \mathbf{e}=\mathbf{S e}
$$

Here, $e_{1}, e_{2}$ is the $X Y$ coordinate and $e_{3}, e_{4}$ is the slope of the wire at point $O$ '. Similarly, $e_{5}, e_{6}$, $e_{7}, e_{8}$ are the $X Y$ coordinate and slope at point $A$ '. The ANCF elastic force is as follows,

$$
\begin{aligned}
\mathbf{F}= & \mathbf{A} 1 \mathbf{e} i_{11}^{2}+\mathbf{A}_{22} \mathbf{e}_{12}^{2}+\left(\mathbf{A}_{12}+\mathbf{A}_{21}\right) \mathbf{e} i_{11} i_{12} \\
& +\mathbf{B}_{11} i_{21}^{2}+\mathbf{B}_{22} \mathbf{e} i_{22}^{2}+\left(\mathbf{B}_{12}+\mathbf{B}_{21}\right) \mathbf{e} i_{21} i_{22}-\mathbf{A}_{1} i_{11}-\mathbf{A}_{2} i_{2} \\
& +\left(\mathbf{e}^{T} \mathbf{A}_{11} \mathbf{e} i_{11}+\frac{1}{2} \mathbf{e}^{T}\left(\mathbf{A}_{\mathbf{1 2}}+\mathbf{A}_{21}\right) \mathbf{e} i_{12}-\mathbf{A}_{1}^{T} \mathbf{e}\right)\left(\frac{\partial i_{11}}{\partial \mathbf{e}}\right)^{T} \\
& +\left(\mathbf{e}^{T} \mathbf{A}_{22} \mathbf{e} i_{12}+\frac{1}{2} \mathbf{e}^{T}\left(\mathbf{A}_{\mathbf{1 2}}+\mathbf{A}_{21}\right) \mathbf{e} i_{11}-\mathbf{A}_{2}^{T} \mathbf{e}\right)\left(\frac{\partial i_{12}}{\partial \mathbf{e}}\right)^{T} \\
& +\left(\mathbf{e}^{T} \mathbf{B}_{11} \mathbf{e} i_{21}+\frac{1}{2} \mathbf{e}^{T}\left(\mathbf{B}_{12}+\mathbf{B}_{\mathbf{2 1}}\right) \mathbf{e} i_{22}\right)\left(\frac{\partial i_{21}}{\partial \mathbf{e}}\right)^{T} \\
& +\left(\mathbf{e}^{T} \mathbf{B}_{\mathbf{2 2}} \mathbf{e} i_{22}+\frac{1}{2} \mathbf{e}^{T}\left(\mathbf{B}_{12}+\mathbf{B}_{\mathbf{2 1}}\right) \mathbf{e} i_{21}\right)\left(\frac{\partial i_{22}}{\partial \mathbf{e}}\right)^{T}
\end{aligned}
$$

Here, $\mathbf{i}_{\mathrm{ij}}$ is the unit for component vector $X Y, \xi=x / l_{e}$ and each factor is defined as follows, 
$\mathbf{A}_{\mathbf{i j}}=\frac{E a}{l_{e}} \int_{0}^{1}\left(\frac{\partial \mathbf{S}_{\mathbf{i}}}{\partial \xi}\right)^{T}\left(\frac{\partial \mathbf{S}_{\mathbf{j}}}{\partial \xi}\right) d \xi$

$\mathbf{B}_{\mathbf{i j}}=\frac{E I}{l_{e}^{3}} \int_{0}^{1}\left(\frac{\partial \mathbf{S}_{\mathbf{i}}}{\partial \xi}\right)^{T}\left(\frac{\partial \mathbf{S}_{\mathbf{j}}}{\partial \xi}\right) d \xi$

$\mathbf{A}_{\mathbf{i}}=E a \int_{0}^{1}\left(\frac{\partial \mathbf{S}_{\mathbf{i}}}{\partial \xi}\right) d \xi$

$(i, j=1,2)$

Thus, ANCF equation of motion would be as follows,

$\mathbf{M e ̈}=\mathbf{Q}-\mathbf{F}$

\subsection{ANCF with damping force}

The elastic force in the ANCF is presented in equation (8). The amount of the wire deformation is expressed in a complex formula. An easy equation is proposed to represent the motion of the small deformation in the stringing wire. This has led to shortening computational time in the wire simulation ${ }^{(13)}$. The wire height and length are considered for the shape function and the impact of the moment of inertia is derived from the mass matrix. Moreover, the simple equation of axial strain is represented by elastic force and bending deformation. The axial strain energy and bending deformation energy are derived from the damping force method at the same time.

The axial strain is assumed to be very small, and the shape function $\mathbf{S}$ with consideration of wire height is shown in the following function,

$\mathbf{S}_{\mathbf{a}}=\left(\mathbf{S}+y\left[\begin{array}{cc}0 & -1 \\ 1 & 0\end{array}\right] \frac{d \mathbf{S}}{d x}\right)$

Here, $y$ is the height of the wire. The elastic force is transformed by the axial strain energy and bending deformation energy.

$U_{l}=\frac{1}{2} \int_{0}^{l_{e}} E a \varepsilon^{2} d x$

$U_{t}=\frac{1}{2} \int_{0}^{l e} E I \kappa^{2} d x$

And the elastic force is derived as follows,

$\mathbf{F}_{\mathbf{l}}=\left(\frac{\partial U_{l}}{\partial \mathbf{e}}\right)^{T}$

$\mathbf{F}_{\mathbf{t}}=\left(\frac{\partial U_{t}}{\partial \mathbf{e}}\right)^{T}$

Here, $\varepsilon$ and $\kappa$ are strain and curvature derivative of arbitrary point $\mathrm{x}$ from point $O$ ' on the wire. The stringing motion of the flexible wire is a significant impact, due to damping property which depending on the material of the wire. However, ANCF does not consider 
the damping parameter of the wire.

Rayleigh damping equation has been considered in the derivation of ANCF model with damping force ${ }^{(13)}$. In previous research, the damping parameter identification procedure had never been proposed. In this study, an easy damping parameter identification experiment is introduced in conjunction with drag coefficient parameter and the formulation of air resistance ${ }^{(14)}$. The damping force, depending on the material of the wire, is produced by the axial strain energy and bending deformation energy,

$U_{I D}=\frac{1}{2} \int_{0}^{l e} D a \dot{\varepsilon}^{2} d x$

$U_{t D}=\frac{1}{2} \int_{0}^{l e} D I \dot{\kappa} d x$

And the damping force is derived as follows,

$$
\begin{aligned}
& \mathbf{F}_{\mathbf{I D}}=\left(\frac{\partial U_{l D}}{\partial \dot{\mathbf{e}}}\right)^{T} \\
& \mathbf{F}_{\mathbf{t D}}=\left(\frac{\partial U_{t D}}{\partial \dot{\mathbf{e}}}\right)^{T}
\end{aligned}
$$

Where, $D$ is a newly introduced damping parameter. The drag force acting on the wire is derived from the following formulas.

$$
\begin{aligned}
& \mathbf{Q}_{\mathbf{D x}}=C_{D} a_{x} \frac{\rho_{a} \cdot v_{x}^{2}}{2}\left[\begin{array}{llllllll}
0 & -\frac{1}{2} & 0 & -\frac{l_{e}}{12} & 0 & -\frac{1}{2} & 0 & \frac{l_{e}}{12}
\end{array}\right]^{T} \\
& \mathbf{Q}_{\mathbf{D y}}=C_{D} a_{y} \frac{\rho_{a} \cdot v_{y}^{2}}{2}\left[\begin{array}{llllllll}
-\frac{1}{2} & 0 & -\frac{l_{e}}{12} & 0 & -\frac{1}{2} & 0 & \frac{l_{e}}{12} & 0
\end{array}\right]^{T}
\end{aligned}
$$

Thus, the damping force formulated ANCF equation of motion is as follows.

$\mathbf{M}_{\mathbf{a}} \ddot{\mathbf{e}}=\mathbf{Q}-\mathbf{F}_{\mathbf{l}}-\mathbf{F}_{\mathbf{t}}-\mathbf{F}_{\mathbf{I D}}-\mathbf{F}_{\mathbf{t}}+\mathbf{Q}_{\mathbf{D x}}+\mathbf{Q}_{\mathbf{D y}}$

\section{Comparison Analysis}

In the previous research of multibody dynamics, the comparison between numerical analysis and experimental result for validation purpose is small. Therefore, in this study, free vibration experimental procedure is conducted and compared with three types of numerical analyses where each analysis is examined for its effectiveness.

\subsection{Experiment}

In the experimental procedure, a wire is hanged vertically and attached with various tensions. Figure 2 shows the schematic diagram of the experimental apparatus. Since copper wire is used in the actual system, the same copper based material is used in this experiment with diameter $3 \mathrm{~mm}$. The experiment is performed under two different conditions. In the first condition, the top end is fixed with rod end bearing. Therefore, the rod can rotate freely but the $x y$-displacement is restrained. The bottom of the rod is fixed with two bearings, so that it only moves on the $y$-direction (Fig. 2(a)). In the second condition (Fig. 2 (b)), the top 
end is fixed with $15 \mathrm{~mm}$ vise clamp. As a result, the rotation and $x y$-displacement are restrained. The bottom part is in the same condition as in the first. The tension load is attached at the bottom of the rod. Initial displacement is given to the rod and as the free vibration begins, laser displacement sensor is used to measure the amplitude to the vibration. The tension is varied from 0 to $70 \mathrm{~N}$ and the characteristic of the frequency is measured. The result of the experiment is shown in Fig. 3.

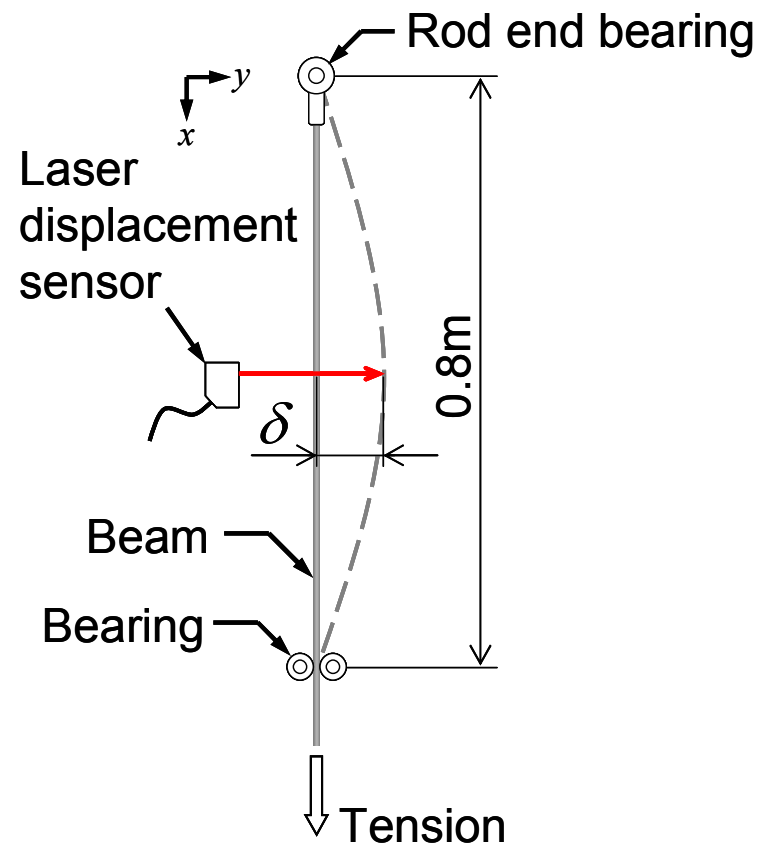

(a)

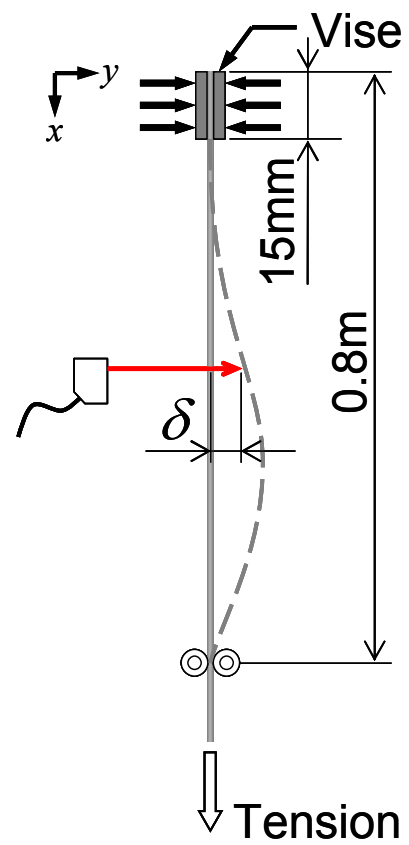

(b) Constraint 2

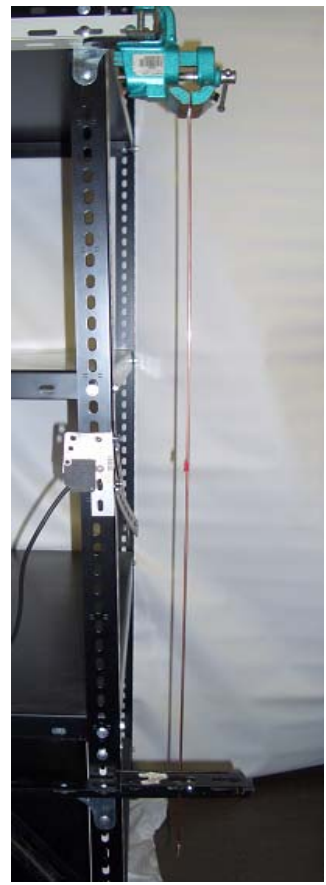

(c) Photo of the experiment setup

Fig. 2 Experiment setup 


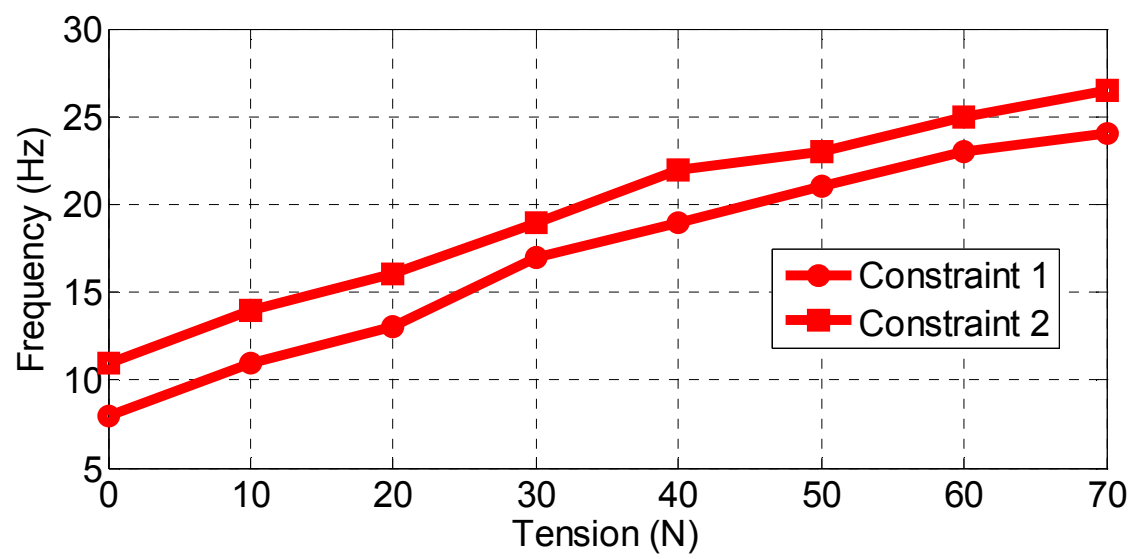

Fig. 3 Experiment result

\subsection{Numerical model}

The wire is divided into $\mathrm{n}$ number of elements where it started from the top of the wire with element $i=1$, and end at the bottom with element $i=n$. Absolute coordinate system started at the top of the wire with $y$-axis for vertical and $x$-axis for horizontal. In the condition 1 , the $x y$-displacement at the top of the wire is restrained with high stiffness spring, but it can rotate freely. Meanwhile the restrain at the bottom of the wire using spring restraints is only on the $x$ displacement. In condition 2, the top of the wire is restrained from rotation and $x y$-displacement using restraint spring represented by $15 \mathrm{~mm}$ clamp vise. The same condition set at the bottom of the wire where only $x$ displacement is restrained. The motion equation of restraint condition 1 and 2 is represented by restraint matrix $Q_{\text {upper }}$ for the top and $\mathrm{Q}_{\text {lower }}$ for the bottom of the wire.

$\mathbf{M e ̈}=\mathbf{Q}_{\text {upper }}^{i=1}+\mathbf{Q}_{\text {lower }}^{i=n}-\mathbf{F}$

In addition to the nodal coordinates, ANCF and classical FEM elements require the nodal slope. However, it is difficult to express directly the initial shape after deformation into the element nodal coordinate vector. Therefore, the center of the wire is initially pushed with certain force at a certain displacement $\delta_{1}$. As the force is released, the stored energy in the wire will make the wire vibrate freely. The shape before and after deformation are recorded and the Eigen frequency is derived from the central displacement of the wire.

\subsection{Parameter identification}

Table 1 shows the parameters used for the analysis. Since the number of element $n$ and damping parameter $D$ are unknowns, the parameter identification process is required. The values of the unknown parameters are changed and increased gradually, and the calculation is repeated to identify suitable values to compare with the experiment. The number of elements is investigated within the range of $n=1$ to 40 . Figure 4 shows the relationship between tension and vibration frequency for classical FEM analysis. As the $n$ is increased, the frequency is decreased and the result almost matched the experimental result. In the ANCF method, as shown in Fig. 5, the result starts to converge at $n=20$. In addition to the number of elements, the damping parameter $D$ is introduced in ANCF. The newly improved ANCF is using damping force within its method. The ordinary ANCF and classical FEM do not consider the damping parameter. The finding of $n$ for improved ANCF is shown in Fig. 6 where the result is converge at $n=20$. Since damping parameter is considered in the improved ANCF, it is possible to identify the value of damping parameter $D$. 
Table 1 Parameters of the wire

\begin{tabular}{l|c|c}
\hline Parameter & Symbol & Value \\
\hline Total length & $l$ & $0.8 \mathrm{~m}$ \\
\hline Diameter & $d$ & $3 \times 10^{-3} \mathrm{~m}$ \\
\hline Young modulus & $E$ & $130 \times 10^{9} \mathrm{~N} / \mathrm{m}^{2}$ \\
\hline Mass density & $\rho$ & $8920 \mathrm{~kg} / \mathrm{m}^{3}$ \\
\hline Drag coefficient & $C_{D}$ & 0.8 \\
\hline Air density & $\rho_{a}$ & $1.2929 \mathrm{~kg} / \mathrm{m}^{3}$ \\
\hline Gravity acceleration & $g$ & $9.8 \mathrm{~m} / \mathrm{s}^{2}$ \\
\hline Tension & $T$ & $0-70 \mathrm{~N}$ \\
\hline Number of element & $n$ & Unknown \\
\hline Damping parameter & $D$ & Unknown \\
\hline
\end{tabular}

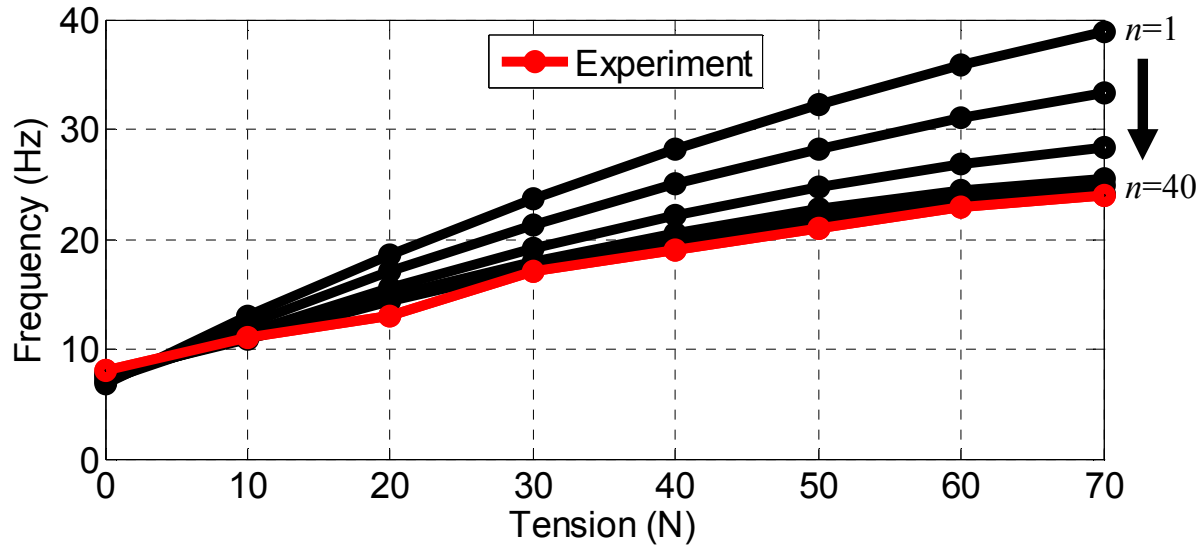

(a) Constraint 1

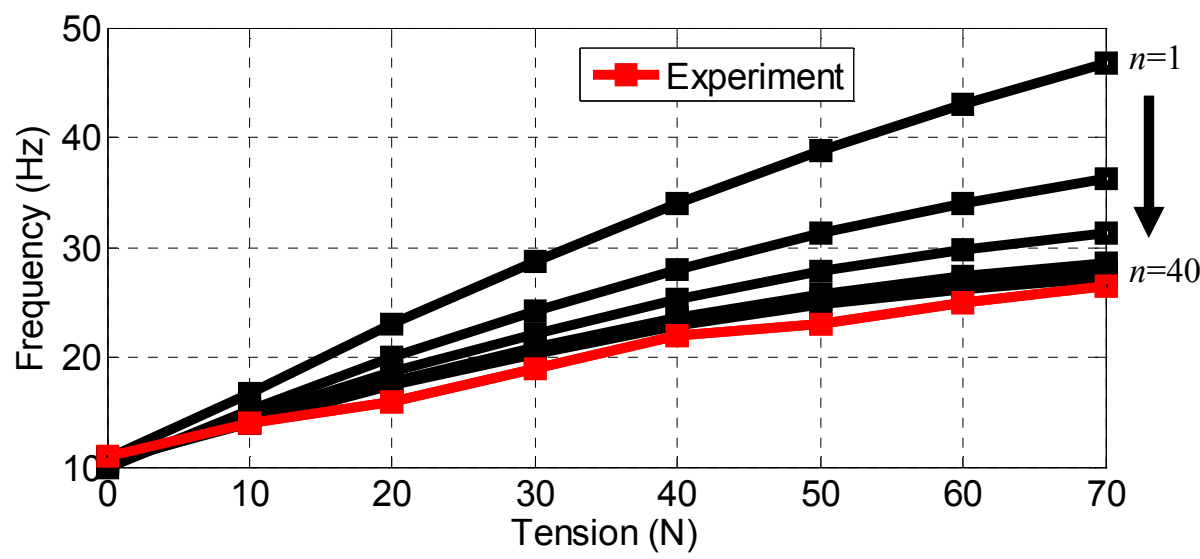

(b) Constraint 2

Fig. 4. Parameter identification using classical FEM 


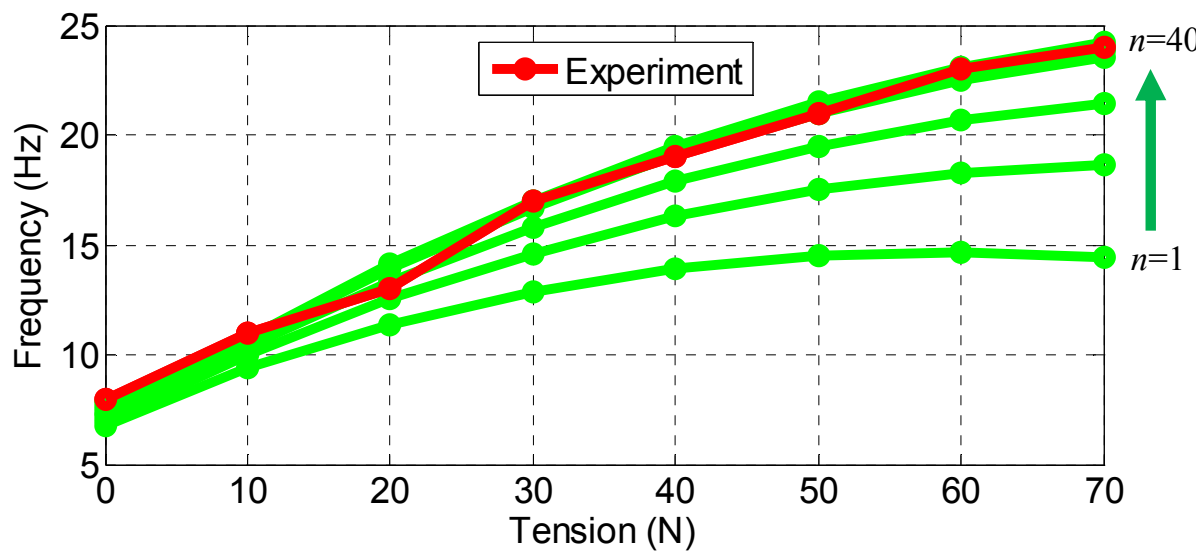

(a) Constraint 1

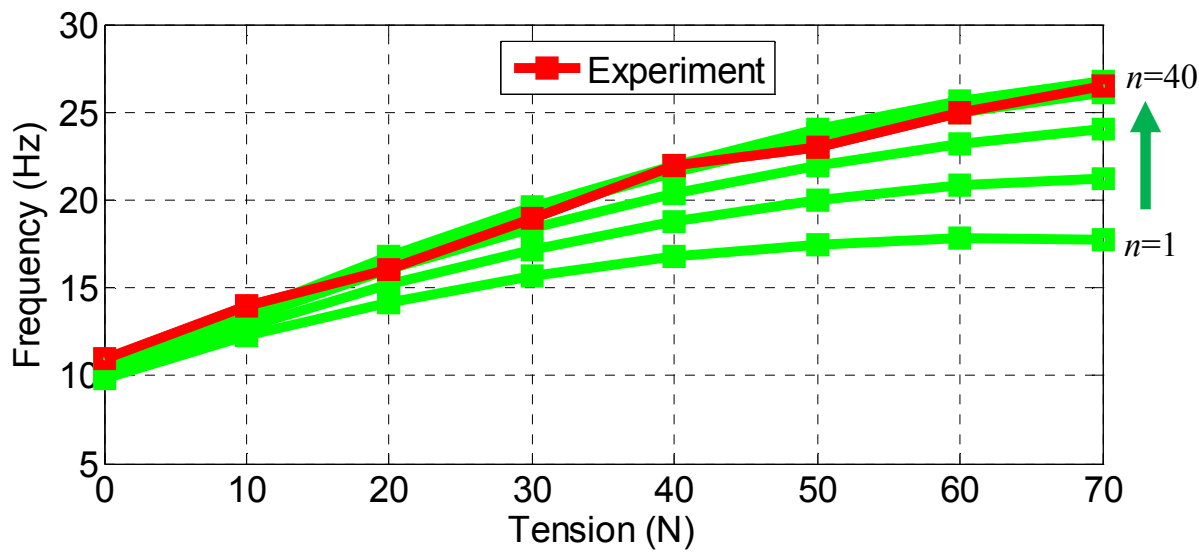

(b) Constraint 2

Fig. 5. Parameter identification using ANCF

The same procedure is applied where the damping parameter is changed and increased gradually in the analysis. The procedure is performed repeatedly and compared with the outcome of experiment. The logarithm damping ratio is calculated from the time history plot, $\varsigma_{e}=0.0055$. After several repeated calculations, the damping ratio and damping parameter are established to have linear relationship. The relationship can be expressed as follows,

$D=\frac{10 \times 10^{7}}{0.017} \varsigma$ e

From the calculation, the damping parameter $D=3.239 \times 10^{7}$ is identified. Figure 7 shows the comparison of damping ratio between analysis and experiment. The result of analysis is matching with experimental result starting at $7.5 \mathrm{~s}$. The unknown parameters for each analysis method are identified and shown in Table 2. In order to evaluate the performance of each method, a convergence characteristic analysis is performed with cost function, $C F_{1}$,

$C F_{1}=\frac{\sum\left|\omega_{x i}-\omega_{m i}\right|}{\sum i}$

Where, $\omega_{x i}$ and $\omega_{m i}$ are the frequency of experiment and numerical results respectively. 


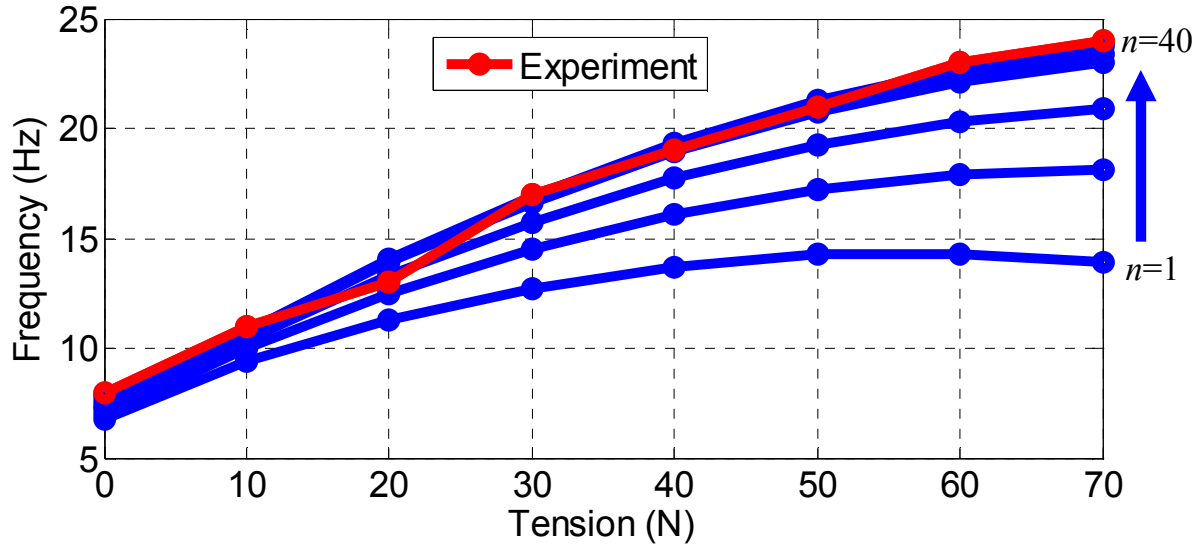

(a) Constraint 1

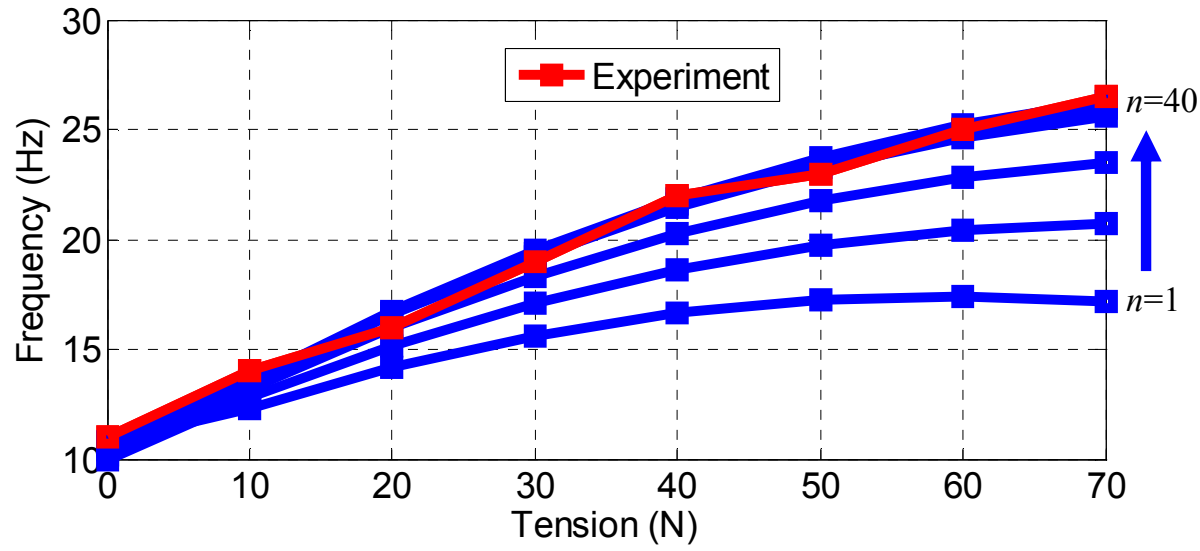

(b) Constraint 2

Fig. 6. Parameter identification using improved ANCF

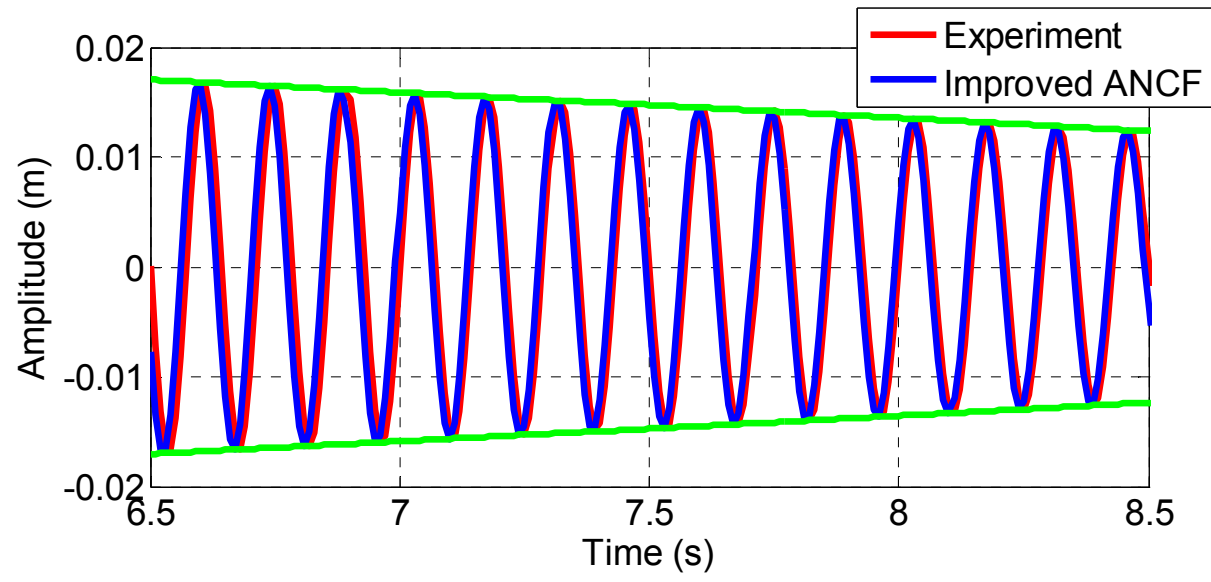

Fig. 7 Comparison of damping ratio

Table 2 Identified parameters

\begin{tabular}{l|c|c}
\hline Method & No. of element & Damping parameter \\
\hline Classical FEM & 30 & - \\
\hline ANCF & 20 & - \\
\hline Improved ANCF & 20 & $3.239 \times 10^{7}$ \\
\hline
\end{tabular}




\subsection{Result}

The frequency characteristic behavior resulting from the applied tension for each method is compared with experiment result in Fig. 8. The results from ANCF and improved ANCF methods are almost the same. This has proven that damping formulation does not have significant effect to the sinusoidal behavior of ANCF method. The classical FEM analysis has higher frequency characteristic compared to ANCF analysis. Using the same number of element $n=20$, ANCF and improved ANCF produced better results compared to classical FEM. The margin of error between experimental result and ANCF and improved ANCF results is lower compared to classical FEM as the applied tension increases. Therefore, the accuracy of ANCF and improved ANCF in motion analysis of overhead wire is improved. The accuracy of one element can reduce the error margin of integration. It is confirmed that the ANCF method is effective for the motion analysis of the wire-like overhead catenary where the tension is acting perpendicularly. The overhead wire is modeled from the analytical result of the characteristic frequency and the accuracy of one element by using the ANCF method with the damping force formulation.

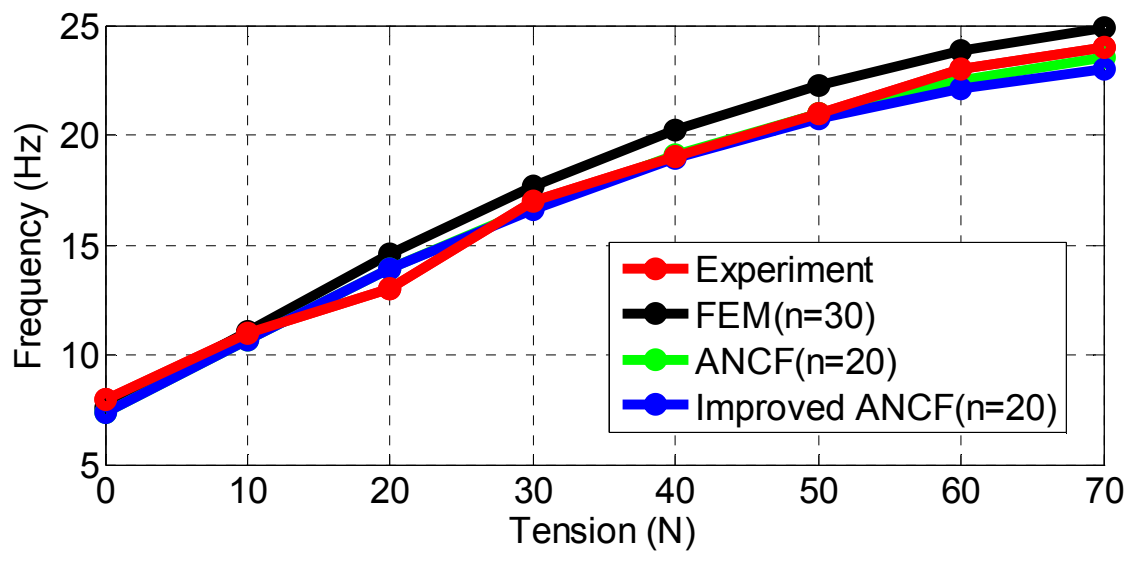

(a) Constraint 1

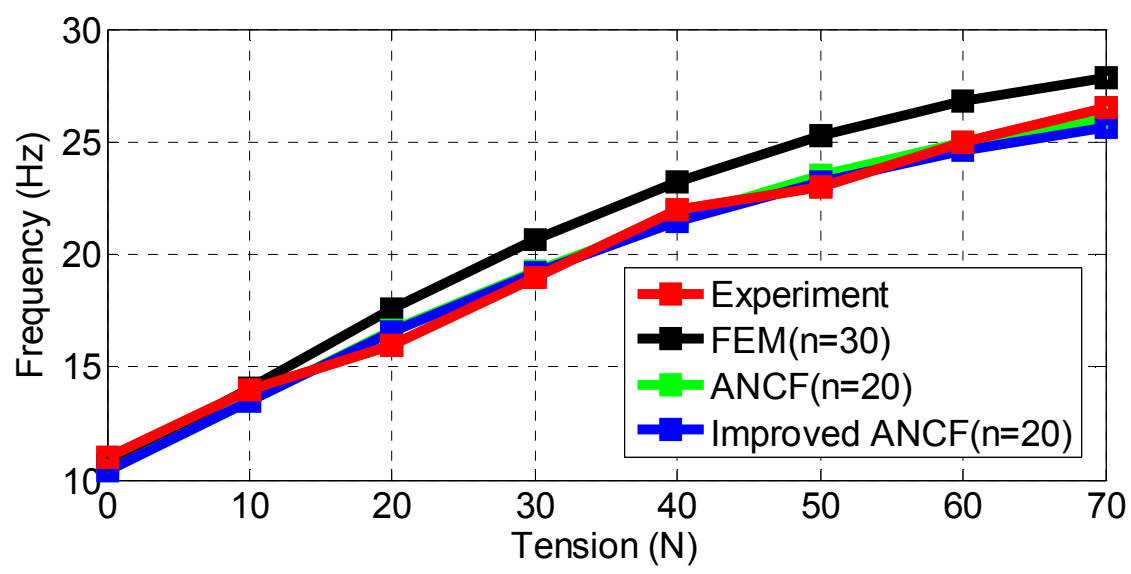

(b) Constraint 2

Fig. 8 Result comparison

Figure 9 shows the convergence characteristic of the simulation results. It is observed that as the number of elements is increased, the result is converging for all modeling methods. Figure 10 illustrates the computational time required for each number of elements. It is logical that as the number of element is increased, the time required for simulation is also increased. Indeed the result is improving, but element performance has certain limitation in regards to computational capacity. 


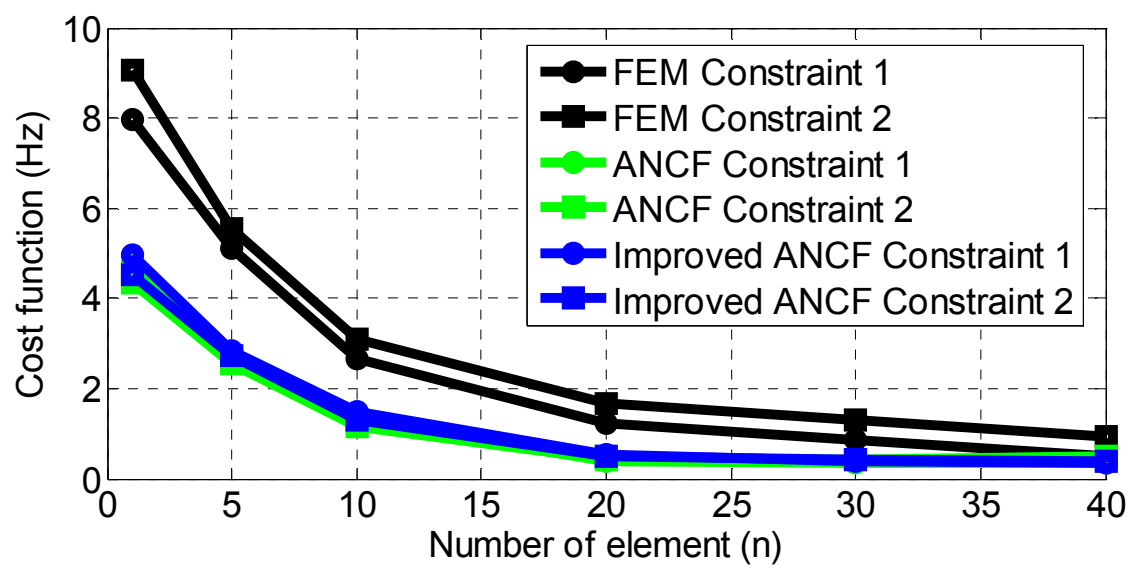

Fig. 9 Convergence characteristic

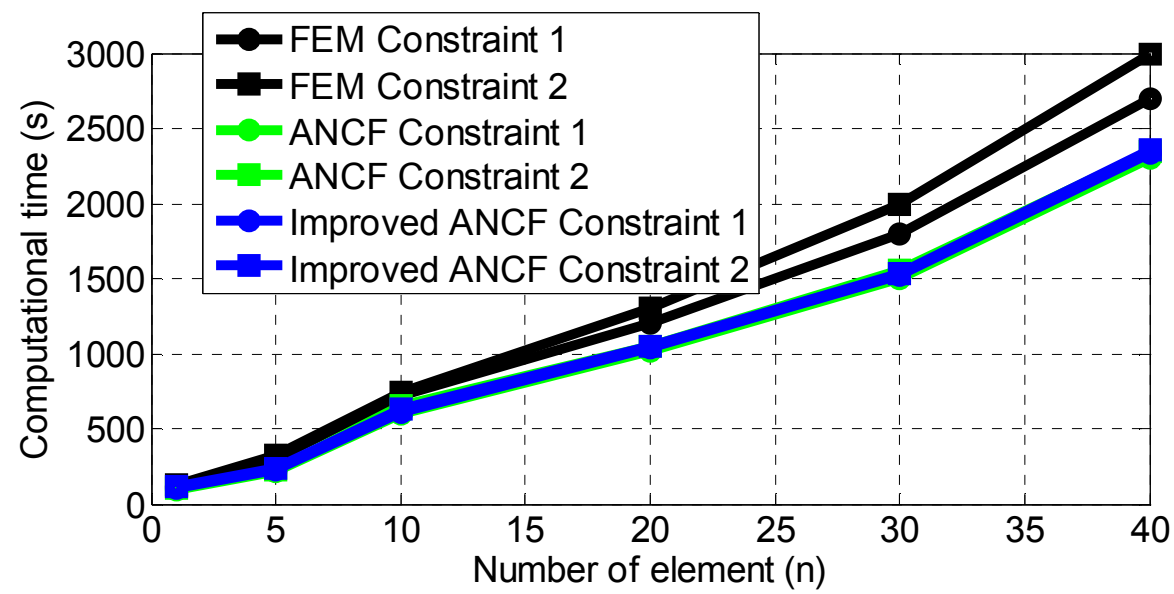

Fig. 10 Computational time

\section{Catenary and Pantograph Interaction Analysis}

\subsection{Element performance analysis}

The previous catenary and pantograph interaction model ${ }^{(9)}$ is used for the element performance analysis. In this analysis, the number of element is changed accordingly to study its convergence solution. Figure 11 shows the relationship between beam element analysis and catenary element analysis. In beam element analysis, the number of element $n$ is changed from 1 to 40 . The same concept is applied for catenary analysis where the number of element per spacing $(E s c)$ is changed from 5 to 20. Each spacing of the catenary is equal to the spacing between 2 holders, in this case, the span length is $50 \mathrm{~m}$, and each span is supported with 10 holders ${ }^{(9)}$. Therefore, the higher the value $E s c$ the smaller the size of one element. The total number of elements for catenary element analysis is shown in Table 3. The second cost function, $C F_{2}$, is used to evaluate the convergence performance.

$C F_{2}=\frac{\sum\left|P_{i}-P_{N}\right|}{\sum i}$

Where, $P_{i}$ is the numerical contact force and $P_{N}$ is the nominal contact force which is equal to $54 \mathrm{~N}$. 


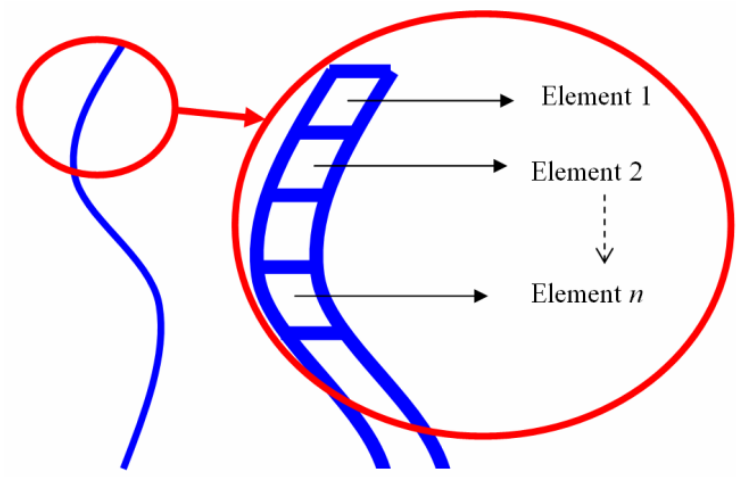

Beam elements

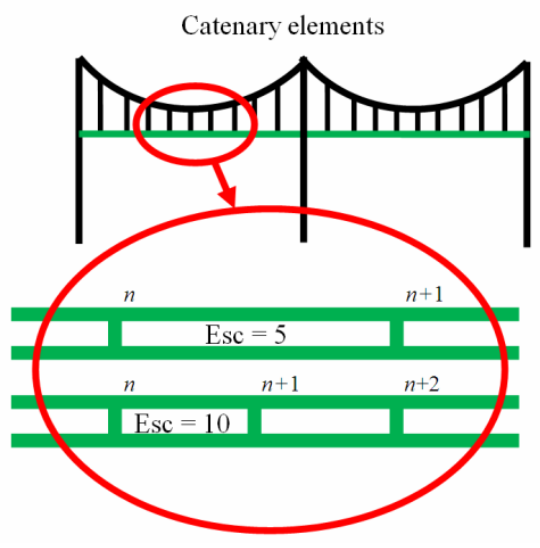

Fig. 11 Element representation

Table 3 Catenary and pantograph interaction simulation parameters

\begin{tabular}{l|c|c}
\hline $\begin{array}{c}\text { Element spacing, } \\
\text { Esc }\end{array}$ & $\begin{array}{c}\text { Total number of } \\
\text { element, } n\end{array}$ & $\begin{array}{c}\text { Contact force matrix } \\
\text { dimensions }\end{array}$ \\
\hline 5 & 100 & $424 \times 424$ \\
\hline 10 & 200 & $844 \times 844$ \\
\hline 15 & 300 & $1264 \times 1264$ \\
\hline 20 & 400 & $1684 \times 1684$ \\
\hline
\end{tabular}

\subsection{Simulation result}

Figure 12 shows the simulation result of the catenary and pantograph interaction at different speeds of 250,300 and $350 \mathrm{~km} / \mathrm{h}$. At the lowest number of elements per spacing, $E s c=5$ (black line), the contact forces seem to have huge discrepancy for all speeds. Since the length for each element is great, the wave propagation motion within the wire affects the displacement and contact force fluctuations by increasing the amplitude of the vibration. This is why at $E s c=5$, the amplitude of each wave is considerably higher. At $E s c=20$ (green line), the vibration of the wire is constrained with small value in length of each element. Therefore, the amplitude of the vibration is considerably lower. This means that the wave propagation motion in the wire does not have enough space to vibrate within small length of each element. Figures 13 and 14 show the convergence characteristic and computational time required for the simulation correspondingly. In Fig 13, the same behavior of convergence is observed when the number of elements is increased. It is validated that the best solution of simulation is at the highest number of elements when element performance simulation is discussed. Nevertheless, higher number of elements requires huge amount of computational calculation. Therefore, selecting good solution in element performance is essential without compromising the computational effort.

\section{Conclusion}

In this study, we have proposed the design of contact wire and pantograph system based on multibody dynamics analysis. The free vibration experiment of a wire with various tensions was conducted and unknown parameters were identified. Comparing three types of analysis, the ANCF with damping force formulation is the most accurate method to model the vibration frequency and damping effect. The validation of the analysis is obtained through the comparison with experimental result. The contact wire is considered as a flexible body modeled by the improved ANCF method. The analysis of contact wire and pantograph contact force variation has shown that the high and low frequency periodic 
contact force are caused by the hanger cycle and span cycle respectively. The bounce of pantograph and displacement of contact wire after the contact phenomenon is a result of relative speed between the operating speed and the wave motion propagation speed. The proposed contact wire and pantograph model can be applied to create controller design and passive parameter control design based on simulation.

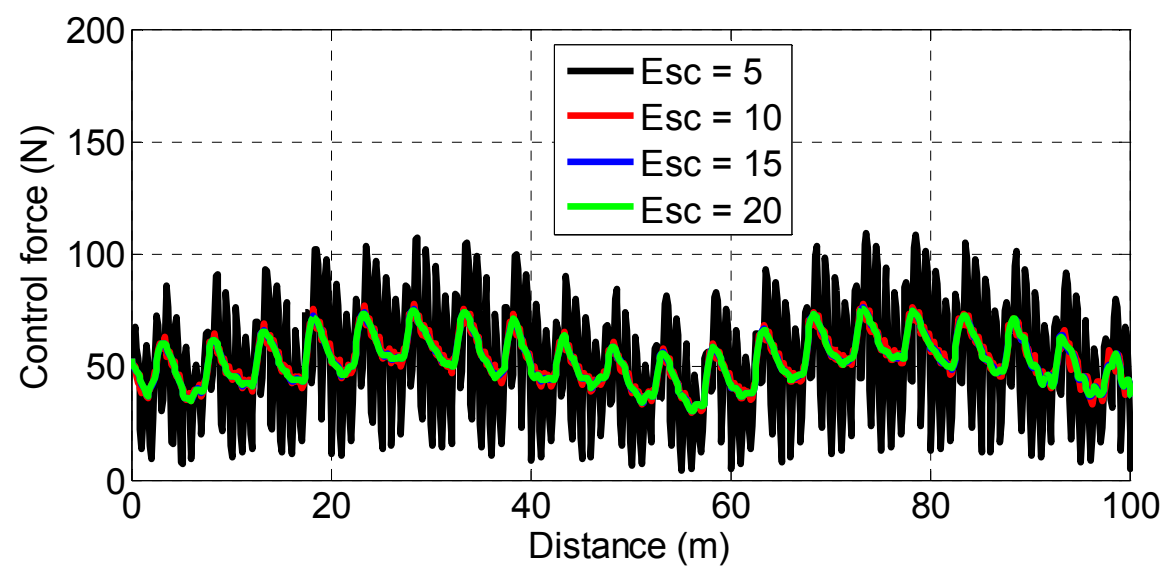

(a) $\mathrm{V}=250 \mathrm{~km} / \mathrm{h}$

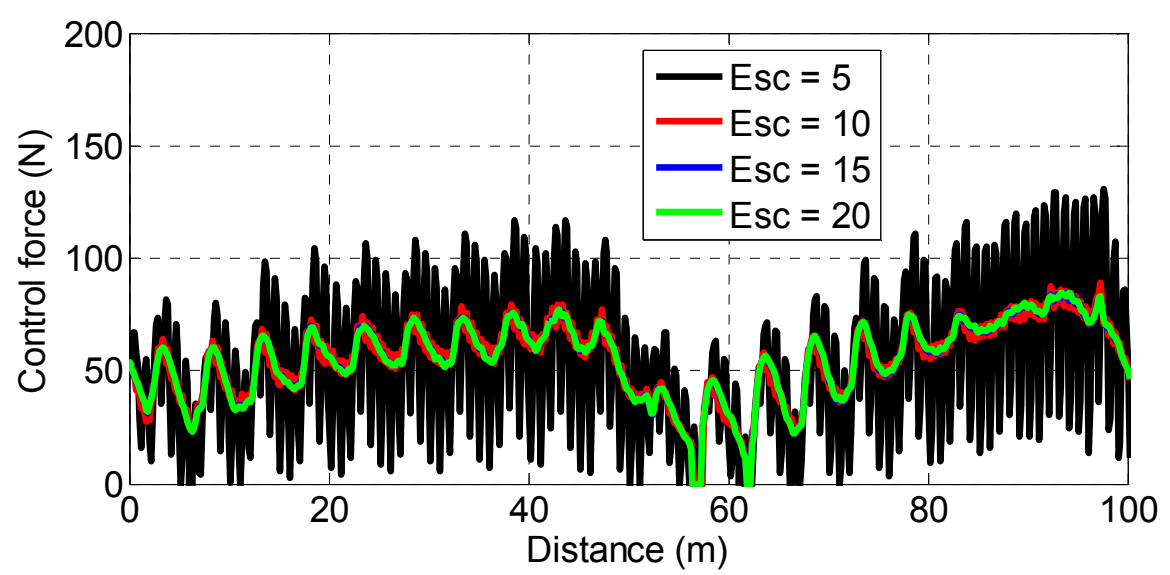

(b) $\mathrm{V}=300 \mathrm{~km} / \mathrm{h}$

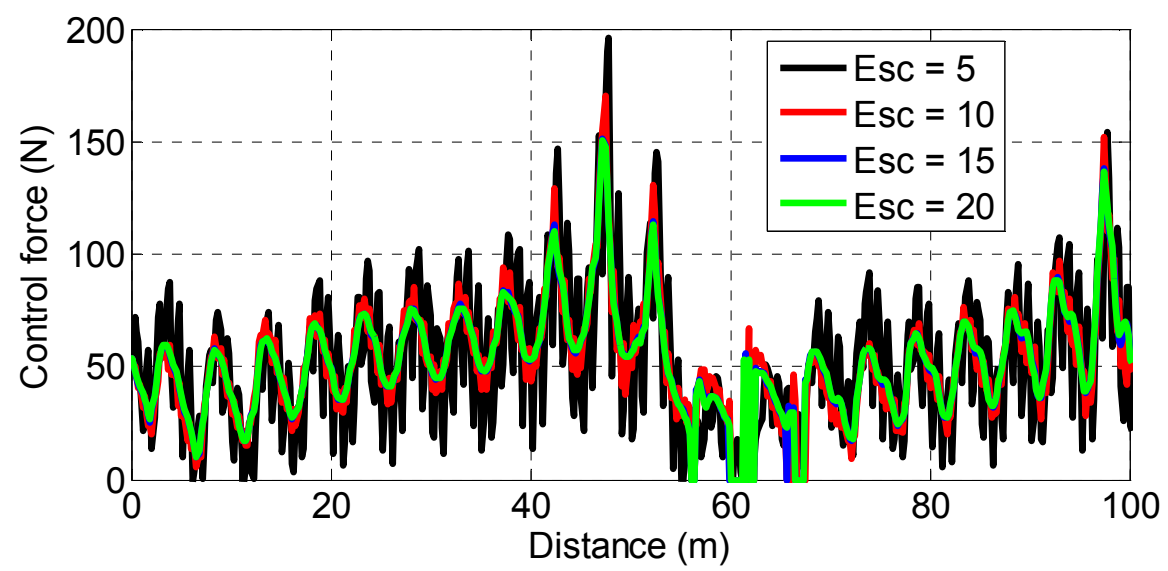

(c) $\mathrm{V}=350 \mathrm{~km} / \mathrm{h}$

Fig. 12 Catenary and pantograph interaction simulation results 


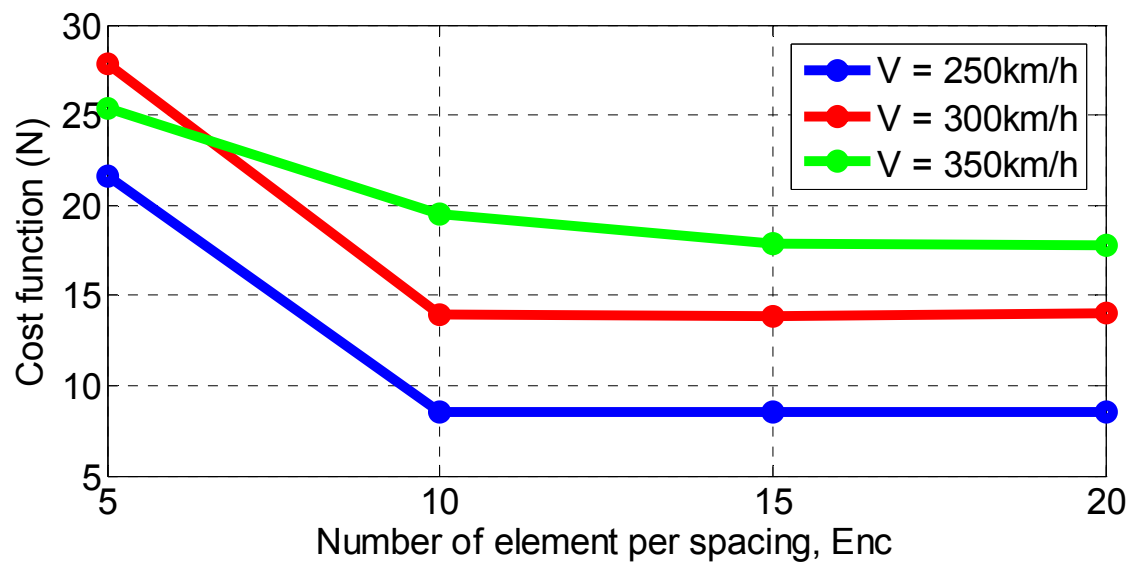

Fig. 13 Catenary and pantograph interaction simulation convergence characteristic

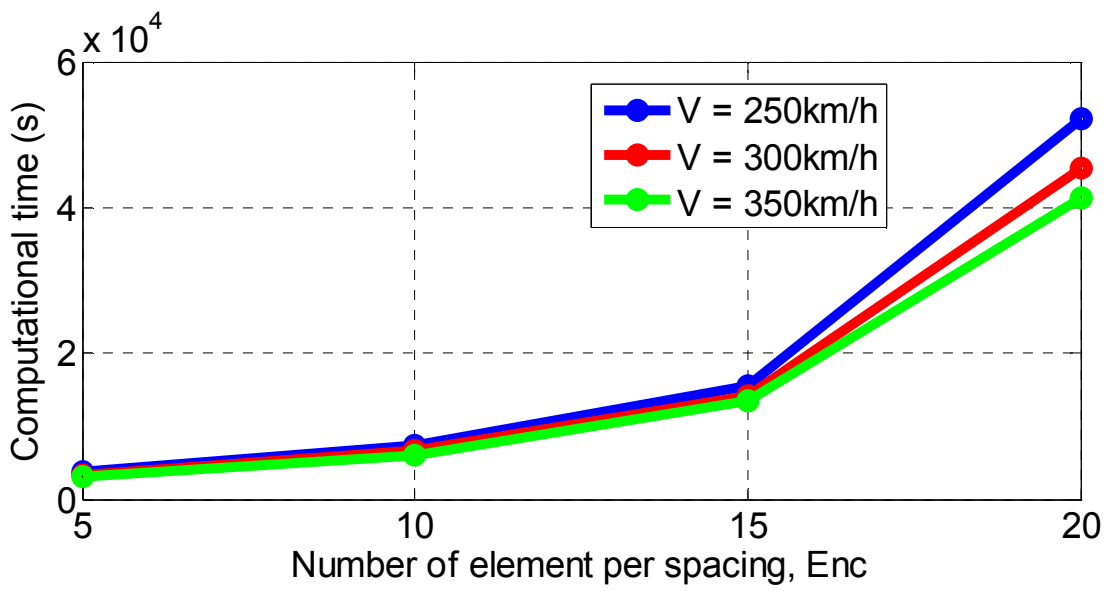

Fig. 14 Catenary and pantograph interaction simulation computational time

\section{Acknowledgement}

The author (Mohd Azman Abdullah) would like to acknowledge the financial support from the Ministry of Higher Education of Malaysia and Universiti Teknikal Malaysia Melaka under the SLAI financial scheme.

\section{References}

(1) Kusumi, S., Fukutani, T. and Nezu, K., Diagnosis of Overhead Contact Line Based on Contact Force, QR of RTRI, Vol.47, No.1, pp.36-45 (2006).

(2) Poetsch, G., Evans, J., Meisinger, R., Kortum, W., Baldauf, W., Veitl, A. and Wallascheck, J., Pantograph/Wire Dynamics and Control, Vehicle System Dynamics Measurement, and Control, Vol.28, pp.159-195 (1997).

(3) Manabe, K., High-Speed Contact Performance of a Wire-Pantograph System, JSME International Journal, Vol.32, pp.31-40 (1989).

(4) Yagi, T., Stensson, A. and Hardell, C., Simulation and Visualization of the Dynamic Behaviour of an Overhead Power System with Contact Breaking, Vehicle System Dynamics, Vol.25, pp.31-49 (1996).

(5) Arnold, M. and Simeon, B., Pantograph and Wire Dynamics - A Benchmark Problem and Its Numerical Solution, Applied Numerical Mathematics, Vol.34, pp.345-362 (2000). 
(6) Wu, T., X. and Brennan, M. J., Basic Analytical Study of Pantograph-Wire System Dynamics, Vehicle System Dynamics, Vol.30, pp.443-456 (1998).

(7) Zhai, W., M. and Cai, C. B., Effect of Locomotive Vibrations on Pantograph-Wire System Dynamics, Vehicle System Dynamics, Vol.29, pp.47-58 (1998).

(8) Lesser, M., Karlsson, L. and Drugge, L., An Interactive Model of a Pantograph-Wire System, Vehicle System Dynamics, Vol.25, pp.397-412 (1996).

(9) Abdullah, M. A., Michitsuji, Y., Nagai, M. and Miyajima, N., Integrated Simulation between Flexible Body of Wire and Active Control Pantograph for Contact Force Variation Control, Journal of Mechanical Systems for Transportation and Logistics, Vol. 3, No. 1, pp.166-177, 2010.

(10) Seo, J-H., Kim, S-W., Jung, I-H., Park, T-W., Mok, J-Y., Kim, Y-G. and Chai, J-B.: Dynamic Analysis of a Pantograph-Catenary System using Absolute Nodal Coordinates, Vehicle System Dynamic, Vol.44, No.8, 615-630 (2006).

(11) Sugiyama, H., Escalona, J. L., and Shabana, A. A.: Formulation of Three-Dimensional Joint Constraints Using the Absolute Nodal Coordinates, Nonlinear Dynamic, Vol.31, No.2, 167-195 (2003).

(12) Shabana, A. A., Dynamics of Multibody Systems ( $3^{\text {rd }}$ Edition), Cambridge, Cambridge University Press, 2005.

(13) Takahashi, Y. and Shimizu, N., Study on the Elastic Force for the Deformed Wire by means of the Absolute Nodal Coordinate Multibody Dynamics Formulation, Transactions of the Japan Society of Mechanical Engineers, Series C., Vol.67, No.655, pp.626-632 (2001).

(14) Miyajima, N., Michitsuji, Y. and Takehara, S., Swing-up Control of Mass Body Interlinked Tether, Transactions of the Japan Society of Mechanical Engineers, Series C., Vol.73, No.735, pp.2955-2961 (2007). 